\title{
Spectral Sum Rules of the Dirac Operator and Partially Quenched Chiral Condensates
}

\author{
P.H. Damgaard and K. Splittorff \\ The Niels Bohr Institute \\ Blegdamsvej 17 \\ DK-2100 Copenhagen $\varnothing$ \\ Denmark
}

June 18, 2021

\begin{abstract}
Exploiting Virasoro constraints on the effective finite-volume partition function, we derive generalized Leutwyler-Smilga spectral sum rules of the Dirac operator to high order. By introducing $N_{v}$ fermion species of equal masses, we next use the Virasoro constraints to compute two (low-mass and large-mass) expansions of the partially quenched chiral condensate through the replica method of letting $N_{v} \rightarrow 0$. The low-mass expansion can only be pushed to a certain finite order due to de Wit-'t Hooft poles, but the large-mass expansion can be carried through to arbitrarily high order. Results agree exactly with earlier results obtained through both Random Matrix Theory and the supersymmetric method.
\end{abstract}

NBI-HE-99-52

hep-th/9912146 


\section{Introduction}

The exact spectral sum rules for the Dirac operator in finite-volume gauge theories derived by Leutwyler and Smilga [1] and later by Smilga and Verbaarschot [2] have sparked a huge activity in the field. It has turned out that there is an exact relation between universality classes in Random Matrix Theories and the three main ways in which chiral symmetry can break spontaneously, depending on the gauge group and the representation of the fermion fields [3, 4, 5]. The Random Matrix Theory formulation brings a completely different set of techniques into play, and much has been learned from this approach. One of the main efforts recently has nevertheless been to see how all the results obtained from Random Matrix Theory can be derived directly from the effective partition function. One of the first steps in this direction was a set of very compact relations that expressed microscopic spectral correlators (including the microscopic spectral density itself) directly in terms of effective partition functions with additional quark species [6]. More recently, many of these results have been derived directly from the effective field theory [7]. The idea has been to compute the (partially) quenched chiral condensate and higher chiral susceptibilities through an appropriately extended effective Lagrangian, and from this derive the microscopic spectral correlators. As the technique of ref. [7] makes use of additional quark species, of which half are bosonic and the others are fermionic, this extends the flavor symmetry to a super Lie group at intermediate steps. For this reason it is commonly known as the supersymmetric method (although it, as applied, has nothing to do with space-time supersymmetry).

The purpose of this paper is to explore another technique that can be used to derive the partially quenched chiral condensate. This is based on the so-called replica method in which one extends the theory with $N_{v}$ additional fermionic species, and takes the limit $N_{v} \rightarrow 0$ at the end of the calculation. The replica method is known in other contexts to be often problematical [8] (see however also the recent discussion on this issue [9]), but we shall find no fundamental difficulties with this technique in the present context.

Let us first outline how the replica method can be used to compute partially quenched averages in QCD. Consider the QCD partition function with $N_{f}$ physical quark fields and $N_{v}$ additional quark fields of degenerate masses $m_{v}$. We restrict ourselves to gauge field sectors of fixed topological charge $\nu$, which we for simplicity from now on take to be non-negative:

$$
\mathcal{Z}_{\nu}^{\left(N_{f}+N_{v}\right)}=\left(\prod_{f=1}^{N_{f}} m_{f}^{\nu}\right) m_{v}^{N_{v} \nu} \int[d A]_{\nu} \operatorname{det}^{\prime}\left(i \not D-m_{v}\right)^{N_{v}} \prod_{f=1}^{N_{f}} \operatorname{det}^{\prime}\left(i \not D-m_{f}\right) e^{-S_{Y M}[A]}
$$

where the determinants are taken over non-zero modes only. This can be considered as an average over gauge fields (and physical fermions, here already integrated out) of $N_{v}$ identical replicas of the fermionic partition function

$$
\mathcal{Z}_{v} \equiv \int d \bar{\psi} d \psi \exp \left[\int d^{4} x \bar{\psi}\left(i \not D-m_{v}\right) \psi\right]
$$

in the sense that

$$
\mathcal{Z}_{\nu}^{\left(N_{f}+N_{v}\right)}=\left(\prod_{f=1}^{N_{f}} m_{f}^{\nu}\right) \int[d A]_{\nu} \prod_{f=1}^{N_{f}} \operatorname{det}^{\prime}\left(\not \not D-m_{f}\right)\left[\mathcal{Z}_{v}\right]^{N_{v}} e^{-S_{Y M}}
$$

\footnotetext{
${ }^{1}$ Or replica "trick", a name that suggests magic and trickery. We prefer the more neutral terminology.
} 
In condensed matter physics this (unnormalized) average is conventionally denoted by a bar:

$$
\mathcal{Z}_{\nu}^{\left(N_{f}+N_{v}\right)}=\overline{\left[\mathcal{Z}_{v}\right]^{N_{v}}}
$$

The subscript $v$ will throughout denote "valence", and the additional quarks are thus valence quarks, a terminology borrowed from lattice gauge theory. Obviously, if we let $N_{v}=0$ we simply recover the original QCD partition function. For $N_{f}+N_{v}$ not too large, the theory is presumed to undergo, in the chiral limit of massless quarks, spontaneous chiral symmetry breaking according to the usual pattern $\mathrm{SU}\left(N_{f}+N_{v}\right) \times \mathrm{SU}\left(N_{f}+N_{v}\right) \rightarrow \mathrm{SU}\left(N_{f}+N_{v}\right)$. Taking the limit $N_{v} \rightarrow 0$ must therefore incorporate some kind of analytic continuation in $n$ of the Lie group $\mathrm{SU}(n)$.

For any fixed $N_{v}$ we can compute observables in the extended theory by viewing the partition function as the generating function of the $n$-point functions of $\bar{\psi} \psi$. We shall here focus on just the chiral condensate itself, and only on the chiral condensate of the additional fermionic copies. As we will take $N_{v} \rightarrow 0$, this becomes the partially quenched condensate in the theory with $N_{f}$ physical fermions. We define this partially quenched (mass-dependent) chiral condensate by

$$
\frac{\Sigma_{\nu}\left(\mu_{v},\{\mu\}\right)}{\Sigma} \equiv \lim _{N_{v} \rightarrow 0} \frac{1}{N_{v}} \frac{\partial}{\partial \mu_{v}} \ln \mathcal{Z}_{\nu}^{\left(N_{f}+N_{v}\right)}
$$

where $\Sigma$ is the physical infinite-volume chiral condensate in the theory with $N_{f}$ fermions, $\mu_{j} \equiv m_{j} V \Sigma$ and similarly $\mu_{v} \equiv m_{v} V \Sigma$. Treating $N_{v}$ as a parameter that is not restricted to be integer (which is permitted by the representation of the partition function in eq. (1.1)), we can make a Taylor expansion in $N_{v}$ :

$$
\mathcal{Z}_{\nu}^{\left(N_{f}+N_{v}\right)}=\mathcal{Z}_{\nu}^{\left(N_{f}\right)}+\left.N_{v} \frac{\partial}{\partial N_{v}} \mathcal{Z}_{\nu}^{\left(N_{f}+N_{v}\right)}\right|_{N_{v}=0}+\ldots
$$

and thus write, equivalently,

$$
\frac{\Sigma_{\nu}\left(\mu_{v},\{\mu\}\right)}{\Sigma}=\left.\left[\mathcal{Z}_{\nu}^{\left(N_{f}\right)}\right]^{-1} \frac{\partial}{\partial N_{v}} \frac{\partial}{\partial \mu_{v}} \mathcal{Z}_{\nu}^{\left(N_{f}+N_{v}\right)}\right|_{N_{v}=0}
$$

The partially quenched chiral condensate is a particularly convenient quantity for lattice gauge theory simulations, and comparisons with theory have already been made both in the topologically trivial sector [10] and, very recently, in sectors of non-vanishing topological charge $\nu$ [11]. We note here that higher-order partially quenched chiral susceptibilities, which are also readily studied by Monte Carlo simulations, can be derived in a completely analogous manner. For a chiral $k$-point function one simply needs to introduce $N_{v 1}, \ldots, N_{v k}$ sets of different additional species (each of $N_{v i}$ 'th degenerate masses $\mu_{v i}$ ), and take the combined limit of all $N_{v i} \rightarrow 0$ in the end, after having performed the required differentiations.

In this paper we will show how the replica method can be applied to the effective finite-volume partition function, and in this way we shall derive analytical series expansions for the partially quenched chiral condensate. As a by-product of the analysis we will get, for free, generalized Leutwyler-Smilga sum rules to very high order. The plan of the paper is as follows. In the next section we review the technique for deriving high-order expansions of the effective partition function on the basis of an iterative sequence of partition function constraints. From this expansion we immediately derive a long list of generalized spectral sum rules of the Dirac operator. In section 3 we turn to the replica method, where we first exploit the same partition function constraints to derive a small-mass expansion for the

\footnotetext{
${ }^{2}$ One can explicitly check that the two derivatives commute in our case.
} 
partially quenched chiral condensate. We also show how a different set of partition function constraints can be used to derive a very high order expansion in large masses. In section 4 we point out that the usual Leutwyler-Smilga spectral sum rules, which by definition are taken with respect to the massless theory, have a natural generalization where the spectral sums are taken with respect to the theory with massive fermions. We derive a series of such massive spectral sum rules, perturbatively expanded in the physical masses. In section 5 we discuss some generalizations to different patterns of spontaneous chiral symmetry breaking, and point out why the present series expansions cannot be used to derive the microscopic spectral density of the Dirac operator itself. In section 6 we briefly list the main conclusions.

\section{Spectral Sum Rules from Virasoro Constraints}

Spectral sum rules of the Dirac operator are derived by comparing the full QCD partition function with the effective low-energy partition function. In the large-volume scaling region $V \ll 1 / m_{\pi}^{4}$, this effective partition function is to leading order [1]

$$
\mathcal{Z}_{\nu}^{\left(N_{f}\right)}=\int_{U \in U\left(N_{f}\right)} d U(\operatorname{det} U)^{\nu} \exp \left[\frac{1}{2} \operatorname{Tr}\left(\mathcal{M} U^{\dagger}+U \mathcal{M}^{\dagger}\right)\right]
$$

where $\mathcal{M}$ is the quark mass matrix rescaled by the space-time volume $V$ times the value $\Sigma$ of the chiral condensate in the chiral limit (and at $\theta=0$ ). We will in fact always take this to be diagonal of entries $\mu_{i}=m_{i} \Sigma V$. In this section we shall restrict ourselves to $N_{f}$ (physical) fermions with no additional quark species, and $N_{v}$ will thus here be taken to be zero from the very beginning. The integration in eq. (2.1) is over the coset of chiral symmetry breaking, here extended from $\mathrm{SU}\left(N_{f}\right)$ to $\mathrm{U}\left(N_{f}\right)$ due to the projection on a sector of fixed topological charge $\nu$. The integral is known in closed form [12],

$$
\mathcal{Z}_{\nu}^{\left(N_{f}\right)}(\{\mu\})=\frac{\operatorname{det} A(\{\mu\})}{\Delta\left(\left\{\mu^{2}\right\}\right)}
$$

where the $N_{f} \times N_{f}$ matrix $A$ is given by

$$
A(\{\mu\})_{i j}=\mu_{i}^{j-1} I_{\nu+j-1}\left(\mu_{i}\right)
$$

$I_{n}(x)$ is a modified Bessel function, and the denominator is given by the Vandermonde determinant of rescaled masses:

$$
\Delta\left(\left\{\mu^{2}\right\}\right) \equiv \prod_{i>j}^{N_{f}}\left(\mu_{i}^{2}-\mu_{j}^{2}\right)=\operatorname{det}_{i, j}\left[\left(\mu_{i}^{2}\right)^{j-1}\right] .
$$

Although the effective partition function is known explicitly, eq. (2.2) is not in a form suitable for the derivation of spectral sum rules of the Dirac operator. To get it into such a form, it is convenient to start with the case $\nu=0$. In that case the left and right invariance of the Haar measure under unitary transformations shows that the partition function depends only on the combination $\mathcal{M}^{\dagger} \mathcal{M}$. A crucial observation of ref. [13, 14] is that the partition function in fact satisfies an infinite set of constraint equations that can be used to determine it uniquely. The precise form of these constraints depend on the chosen variables, and one can use two different sets:

$$
t_{k}^{+} \equiv \frac{1}{4^{k} k} \operatorname{Tr}\left(\mathcal{M}^{\dagger} \mathcal{M}\right)^{k}, \quad k=1,2, \ldots
$$


which is a suitable set for small-mass expansions, and

$$
t_{k}^{-} \equiv-\frac{2^{2 k+1}}{2 k+1} \operatorname{Tr}\left(\left(\mathcal{M}^{\dagger} \mathcal{M}\right)^{-(2 k+1) / 2}\right), \quad k=0,1, \ldots,
$$

which is a suitable set for large-mass expansions (the coefficients in front have been inserted for later convenience). Spectral sum rules of the Dirac operator are derived by means of a small-mass expansion, and we shall therefore begin with the description in terms of the variables $t_{k}^{+}$.

Defining, for $n \geq 1$,

$$
\mathcal{L}_{n}^{+} \equiv N_{f} \frac{\partial}{\partial t_{n}^{+}}+\sum_{k=0}^{\infty} k t_{k}^{+} \frac{\partial}{\partial t_{n+k}^{+}}+\sum_{k=1}^{n-1} \frac{\partial^{2}}{\partial t_{k}^{+} \partial t_{n-k}^{+}},
$$

the partition function (2.10) is found to satisfy 14

$$
\mathcal{L}_{n}^{+} \mathcal{Z}_{0}^{\left(N_{f}\right)}=\delta_{n, 1} \mathcal{Z}_{0}^{\left(N_{f}\right)}
$$

These constraints are consistent in the sense that they satisfy the classical Virasoro algebra

$$
\left[\mathcal{L}_{n}^{+}, \mathcal{L}_{m}^{+}\right]=(n-m) \mathcal{L}_{n+m}^{+},
$$

so that they do not generate new constraints beyond the infinite tower of $\mathcal{L}_{n}^{+}$'s. The constraints are also complete: They determine the partition function uniquely, given the boundary condition that $\mathcal{Z}_{0}^{\left(N_{f}\right)}=1$ for all $t_{k}^{+}=0$.

The small-mass power series for $\mathcal{Z}_{0}^{\left(N_{f}\right)}$ can conveniently be chosen 14

$$
\mathcal{Z}_{0}^{\left(N_{f}\right)}=1+\sum_{M} \sum_{1 \leq k_{1} \ldots \leq k_{M}} C_{N_{f}}(\{k\}) \frac{k_{1} t_{1}^{+} \cdots k_{M} t_{M}^{+}}{\left(k_{1}+\ldots+k_{M}\right) !},
$$

where use has been made of the boundary condition to determine the zeroth order coefficient.

The coefficients $C_{N_{f}}(\{k\})$ are determined uniquely by the Virasoro constraints (2.8). They factorize into a polynomial part, $\hat{C}_{N_{f}}(\{k\})$, and a singular part [14]:

$$
C_{N_{f}}(\{k\}) \equiv \hat{C}_{N_{f}}(\{k\}) \prod_{l=0}^{K(\{k\})-1}\left(N_{f}^{2}-l^{2}\right)^{-1},
$$

where $K(\{k\}) \equiv \sum_{i} k_{i}$. The singular parts will play an important rôle in what follows. Originally noted by de Wit and 't Hooft in the context of strong-coupling expansions in lattice gauge theory [15, these poles are in fact innocuous as far as the expansion of the partition function itself is concerned [16]. What happens is that for (integer and non-vanishing!) values of $N$ the poles only occur in terms that are linearly dependent, and therefore should be combined. After combining them, all poles cancel. This phenomenon becomes obvious when we consider the explicit expansion below.

The coefficients $\hat{C}_{N_{f}}(\{k\})$ have already been computed recursively in ref. 14] to high order, enough to determine the partition function to $8^{\text {th }}$ order in the masses. The restriction to the $\nu=0$ sector can easily be lifted due to flavor-topology duality, which states that

$$
\mathcal{Z}_{\nu}^{\left(N_{f}\right)}\left(\mathcal{M}, \mathcal{M}^{\dagger}\right)=[\operatorname{det}(\mathcal{M})]^{\nu} \mathcal{Z}_{0}^{\left(N_{f}+\nu\right)}\left(\mathcal{M M}^{\dagger}\right)
$$

\footnotetext{
${ }^{3}$ Since $\mathcal{M}^{\dagger} \mathcal{M}$ is a hermitian matrix of size $N_{f} \times N_{f}$, only $N_{f}$ of these variables are independent; this is of no consequence for the subsequent analysis.
} 
where $\mathcal{Z}_{0}^{\left(N_{f}+\nu\right)}\left(\mathcal{M M}^{\dagger}\right)$ is the $\nu=0$ partition function extended with $\nu$ massless quarks [18]. Introducing $N \equiv N_{f}+N_{v}+\nu$ (here with $N_{v}=0$ ) we have

$$
\begin{aligned}
\mathcal{Z}_{\nu}^{\left(N_{f}\right)}= & \operatorname{det}^{\nu}(\mathcal{M})\left(1+\frac{1}{4 N} \operatorname{Tr} \mathcal{M}^{\dagger} \mathcal{M}+\frac{1}{32\left(N^{2}-1\right)}\left(\operatorname{Tr}\left(\mathcal{M}^{\dagger} \mathcal{M}\right)\right)^{2}-\frac{1}{32 N\left(N^{2}-1\right)} \operatorname{Tr}\left(\mathcal{M}^{\dagger} \mathcal{M}\right)^{2}\right. \\
& +\frac{1}{96 N\left(N^{2}-1\right)\left(N^{2}-4\right)} \operatorname{Tr}\left(\left(\mathcal{M}^{\dagger} \mathcal{M}\right)^{3}\right)-\frac{1}{128\left(N^{2}-1\right)\left(N^{2}-4\right)} \operatorname{Tr}\left(\mathcal{M}^{\dagger} \mathcal{M}\right) \operatorname{Tr}\left(\left(\mathcal{M}^{\dagger} \mathcal{M}\right)^{2}\right) \\
& +\frac{N^{2}-2}{384 N\left(N^{2}-1\right)\left(N^{2}-4\right)}\left(\operatorname{Tr}\left(\mathcal{M}^{\dagger} \mathcal{M}\right)\right)^{3}-\frac{5}{1024 N\left(N^{2}-1\right)\left(N^{2}-4\right)\left(N^{2}-9\right)} \operatorname{Tr}\left(\left(\mathcal{M}^{\dagger} \mathcal{M}\right)^{4}\right) \\
& +\frac{2 N^{2}-3}{768 N^{2}\left(N^{2}-1\right)\left(N^{2}-4\right)\left(N^{2}-9\right)} \operatorname{Tr}\left(\mathcal{M}^{\dagger} \mathcal{M}\right) \operatorname{Tr}\left(\left(\mathcal{M}^{\dagger} \mathcal{M}\right)^{3}\right) \\
& +\frac{N^{2}+6}{2048 N^{2}\left(N^{2}-1\right)\left(N^{2}-4\right)\left(N^{2}-9\right)}\left(\operatorname{Tr}\left(\mathcal{M}^{\dagger} \mathcal{M}\right)^{2}\right)^{2} \\
& -\frac{1}{1024 N\left(N^{2}-1\right)\left(N^{2}-9\right)}\left(\operatorname{Tr}\left(\mathcal{M}^{\dagger} \mathcal{M}\right)\right)^{2} \operatorname{Tr}\left(\left(\mathcal{M}^{\dagger} \mathcal{M}^{2}\right)\right) \\
& \left.+\frac{N^{4}-8 N^{2}+6}{6144 N^{2}\left(N^{2}-1\right)\left(N^{2}-4\right)\left(N^{2}-9\right)}\left(\operatorname{Tr}\left(\mathcal{M}^{\dagger} \mathcal{M}\right)\right)^{4}+\ldots\right)
\end{aligned}
$$

We have checked that this expansion is correct to the order given by expanding, for fixed $N_{f}$, the closed expression (2.2) up to that order. (Note that it is not at all simple to rearrange the closed expression (2.2) in terms of an expansion in just powers of traces of the mass matrix; the rearrangements are different for each value of $N_{f}$ ). In this expansion we directly verify the cancellation of de Wit-'t Hooft poles for any of the finite, integer, values involved.

With this high-order expansion of the effective partition function, we can now easily derive a long series of spectral sum rules, following the method of Leutwyler and Smilga 11. Because the method is described in detail in ref. [1], we shall not give details of how these sum rules are extracted, but only quote the results. We rescale as usual the eigenvalues $\lambda_{i}$ of the Dirac operator according to $\zeta_{i} \equiv \lambda_{i} \Sigma V$. Expanding the original partition function (1.1) around the massless theory, and comparing with the above expansion up to $8^{\text {th }}$ order, we then find:

$$
\begin{aligned}
\left\langle\sum_{\zeta_{n}>0} \frac{1}{\zeta_{n}^{2}}\right\rangle & =\frac{1}{4} \frac{1}{N} \\
\left\langle\sum_{\zeta_{n}>0} \frac{1}{\zeta_{n}^{4}}\right\rangle & =\frac{1}{16} \frac{1}{N\left(N^{2}-1\right)} \\
\left\langle\sum_{\zeta_{n}>0} \frac{1}{\zeta_{n}^{6}}\right\rangle & =\frac{1}{32} \frac{1}{N\left(N^{2}-1\right)\left(N^{2}-4\right)} \\
\left\langle\sum_{\zeta_{n}>0} \frac{1}{\zeta_{n}^{8}}\right\rangle & =\frac{5}{256} \frac{1}{N\left(N^{2}-1\right)\left(N^{2}-4\right)\left(N^{2}-9\right)} \\
\left\langle\left(\sum_{\zeta_{n}>0} \frac{1}{\zeta_{n}^{2}}\right)^{2}\right\rangle & =\frac{1}{16} \frac{1}{N^{2}-1} \\
\left\langle\left(\sum_{\zeta_{n}>0} \frac{1}{\zeta_{n}^{2}}\right)^{3}\right\rangle & =\frac{1}{64} \frac{N^{2}-2}{N\left(N^{2}-1\right)\left(N^{2}-4\right)}
\end{aligned}
$$




$$
\begin{aligned}
\left\langle\left(\sum_{\zeta_{n}>0} \frac{1}{\zeta_{n}^{4}}\right)^{2}\right\rangle & =\frac{1}{256} \frac{N^{2}+6}{N^{2}\left(N^{2}-1\right)\left(N^{2}-4\right)\left(N^{2}-9\right)} \\
\left\langle\sum_{\zeta_{m}, \zeta_{n}>0} \frac{1}{\zeta_{m}^{2} \zeta_{n}^{4}}\right\rangle & =\frac{1}{64} \frac{1}{\left(N^{2}-1\right)\left(N^{2}-4\right)} \\
\left\langle\sum_{\zeta_{m}, \zeta_{n}>0} \frac{1}{\zeta_{m}^{2} \zeta_{n}^{6}}\right\rangle & =\frac{1}{256} \frac{2 N^{2}-3}{N^{2}\left(N^{2}-1\right)\left(N^{2}-4\right)\left(N^{2}-9\right)} \\
\left\langle\sum_{\zeta_{k}, \zeta_{m}, \zeta_{n}>0} \frac{1}{\zeta_{k}^{2} \zeta_{m}^{2} \zeta_{n}^{4}}\right\rangle & =\frac{1}{256} \frac{1}{N\left(N^{2}-1\right)\left(N^{2}-9\right)} \\
\left.\sum_{\zeta_{k}, \zeta_{l}, \zeta_{m}, \zeta_{n}>0} \frac{1}{\zeta_{k}^{2} \zeta_{l}^{2} \zeta_{m}^{2} \zeta_{n}^{2}}\right\rangle & =\frac{1}{256} \frac{N^{4}-8 N^{2}+6}{N^{2}\left(N^{2}-1\right)\left(N^{2}-4\right)\left(N^{2}-9\right)}
\end{aligned}
$$

Of these, the relations (2.14), 2.15) and (2.18) have been derived previously [1] (see also ref. [17]). We note that the DeWit-'t Hooft poles make these sum rules divergent for an increasingly larger number of integer $N$-values. This is due to infrared divergences near $\zeta \sim 0$. Just as these poles cancel among different terms in the small-mass expansion, one can form analogous combinations of sum rules that are infrared finite. For example, the de Wit-'t Hooft poles at $N=1$ associated with the terms of order $\mu^{4}$ in eq. (2.13) cancel by combining the third and fourth terms in that expansion. This directly translates into a cancellation between two eigenvalue sum rules from our list:

$$
\left\langle\left(\sum_{\zeta_{n}>0} \frac{1}{\zeta_{n}^{2}}\right)^{2}\right\rangle-\left\langle\sum_{\zeta_{n}>0} \frac{1}{\zeta_{n}^{4}}\right\rangle=\frac{1}{16} \frac{1}{N(N+1)},
$$

a cancellation that was already noticed by Leutwyler and Smilga [1]. Similarly, while sum rules (2.16), (2.19) and (2.21) are individually singular at both $N=1$ and $N=2$, the combination

$$
2\left\langle\sum_{\zeta_{n}>0} \frac{1}{\zeta_{n}^{6}}\right\rangle+\left\langle\left(\sum_{\zeta_{n}>0} \frac{1}{\zeta_{n}^{2}}\right)^{3}\right\rangle-3\left\langle\sum_{\zeta_{m}, \zeta_{n}>0} \frac{1}{\zeta_{m}^{2} \zeta_{n}^{4}}\right\rangle=\frac{1}{64} \frac{1}{N(N+1)(N+2)}
$$

is finite (this is 6 times the $\mathcal{O}\left(1 / \lambda^{6}\right)$ contribution to the partition function). A similar phenomenon persists to all orders.

The massless spectral sum rules (2.14)-2.24) can of course be compared with the chiral Random Matrix Theory result. This is done by averaging the inverse moments according to the universal massless microscopic spectral density 4

$$
\rho_{S}^{\left(N_{f}, \nu\right)}(\zeta)=\frac{|\zeta|}{2}\left[J_{N_{f}+\nu}(\zeta)^{2}-J_{N_{f}+\nu-1}(\zeta) J_{N_{f}+\nu+1}(\zeta)\right]
$$

e.g.

$$
\left\langle\sum_{\zeta_{n}>0} \frac{1}{\zeta_{n}^{2 k}}\right\rangle=\int_{-\infty}^{\infty} d \zeta \frac{\rho_{S}(\zeta)}{\zeta^{2 k}} .
$$

We have explicitly checked a number of the above sum rules, always finding completely agreement with the Random Matrix Theory results. 


\section{The partially quenched chiral condensate}

Let us now extend the effective theory with $N_{v}$ valence quark fields. We are interested in obtaining the partially quenched chiral condensate, not higher order susceptibilities, and therefore take the valence quark masses as degenerate and of size $\mu_{v}=m_{v} \Sigma V$. As mentioned above, the extended partition function is also the generating functional for the $n$-point functions of $\bar{\psi} \psi$ (quenched or unquenched); the partially quenched chiral condensate is obtained through the replica method. Since the partition function for $\nu=0$ only depends on $\mathcal{M}^{\dagger} \mathcal{M}$ and the valence quarks have degenerate masses it is natural to introduce a mass matrix of the physical quarks $M$ so that

$$
\operatorname{Tr}\left(\mathcal{M}^{\dagger} \mathcal{M}\right)=\operatorname{Tr}\left(M^{2}\right)+N_{v} \mu_{v}^{2} .
$$

In short, for the definition of the partially quenched chiral condensate we can take

$$
\frac{\Sigma_{\nu}\left(\mu_{v},\{\mu\}\right)}{\Sigma} \equiv \lim _{N_{v} \rightarrow 0} \frac{1}{N_{v}} \frac{\partial}{\partial \mu_{v}} \ln \mathcal{Z}_{\nu}^{\left(N_{f}+N_{v}\right)} .
$$

The subscript on $\Sigma$ refers to the topological sector, as usual. As we have seen above, it is not particularly helpful to know the exact analytical expression for the (extended) effective partition function $\mathcal{Z}_{\nu}^{\left(N_{f}+N_{v}\right)}$ if one wishes to derive spectral sum rules for the Dirac operator. There a smallmass expansion is required by definition. As for finding the partially quenched chiral condensate using the expression (3.30), no expansion in either large or small masses is required in principle. However, the closed analytical formula (2.2) is unfortunately not directly suitable from our point of view. This is because the $N_{v}$-dependence enters in a quite non-trivial way through the size of the matrix whose determinant needs to be taken. Because there is no simple extension of the analytical expression (2.2) outside integer values of $N_{v}$, we restrict ourselves here to series expansions. We shall in fact be able to carry such expansions through in both the large-mass and small-mass regions.

\subsection{The small-mass expansion}

We begin with the small-mass expansion, because here we already have the needed expansion at hand (see eq. (2.13)). Our first observation is that $N_{v}$ enters in a manner that allows for an analytic continuation once we make use of eq. (3.29). We can thus proceed with the replica method.

Inserting the expansion $(2.13)$ into $(3.30)$ we obtain the partially quenched chiral condensate to $7^{\text {th }}$ order in the masses (truncating the expansion at this order is a matter of choice; we do it because it corresponds to consistently expanding both in the valence quark mass $\mu_{v}$ and the physical fermion masses $\mu_{i}$ to the same order):

$$
\begin{aligned}
\frac{\Sigma_{\nu}\left(\mu_{v},\{\mu\}\right)}{\Sigma}= & \frac{\nu}{\mu_{v}}+\mu_{v}\left[\frac{1}{2 N}+\frac{\operatorname{Tr} M^{2}}{8 N^{2}\left(N^{2}-1\right)}+\frac{\left(\operatorname{Tr} M^{2}\right)^{2}}{8 N^{3}\left(N^{2}-1\right)\left(N^{2}-4\right)}\right. \\
& -\frac{\operatorname{Tr} M^{4}}{16 N^{2}\left(N^{2}-1\right)\left(N^{2}-4\right)}+\frac{15 \operatorname{Tr} M^{6}}{384 N^{2}\left(N^{2}-1\right)\left(N^{2}-4\right)\left(N^{2}-9\right)} \\
& \left.+\frac{3\left(2 N^{2}-3\right)\left(\operatorname{Tr} M^{2}\right)^{3}}{32 N^{4}\left(N^{2}-1\right)^{2}\left(N^{2}-4\right)\left(N^{2}-9\right)}+\frac{3\left(3-2 N^{2}\right) \operatorname{Tr} M^{2} \operatorname{Tr} M^{4}}{32 N^{3}\left(N^{2}-1\right)^{2}\left(N^{2}-4\right)\left(N^{2}-9\right)}+\ldots\right] \\
& -\mu_{v}^{3}\left[\frac{1}{8 N\left(N^{2}-1\right)}+\frac{\operatorname{Tr} M^{2}}{8 N^{2}\left(N^{2}-1\right)\left(N^{2}-4\right)}\right.
\end{aligned}
$$




$$
\begin{aligned}
& +\frac{\left(6 N^{2}-9\right)\left(\operatorname{Tr} M^{2}\right)^{2}}{32 N^{3}\left(N^{2}-1\right)^{2}\left(N^{2}-4\right)\left(N^{2}-9\right)} \\
& \left.-\frac{3\left(3 N^{2}-7\right) \operatorname{Tr} M^{4}}{128 N^{2}\left(N^{2}-1\right)^{2}\left(N^{2}-4\right)\left(N^{2}-9\right)}+\ldots\right] \\
& +\mu_{v}^{5}\left[\frac{1}{16 N\left(N^{2}-1\right)\left(N^{2}-4\right)}+\frac{15 \operatorname{Tr} M^{2}}{128 N^{2}\left(N^{2}-1\right)\left(N^{2}-4\right)\left(N^{2}-9\right)}+\ldots\right] \\
& -\mu_{v}^{7} \frac{5}{128 N\left(N^{2}-1\right)\left(N^{2}-4\right)\left(N^{2}-9\right)}+\ldots
\end{aligned}
$$

where $N=N_{f}+\nu$ (since the $N_{v} \rightarrow 0$ limit has already been taken). Note that the "topological" term $\nu / \mu_{v}$ comes out trivially from the $(\operatorname{det} \mathcal{M})^{\nu}$-factor in front, as it should.

Is this expansion correct? There are by now two independent ways to check it: Comparing with Random Matrix Theory, and comparing with the supersymmetric method of partially quenched Lagrangians. Before proceeding with such comparisons, let us however first note an obvious feature of the above expansion. In contrast to the small-mass expansion of the partition function itself (2.13), there is clearly not going to be a cancellation of the de Wit-'t Hooft poles in the expansion of the partially quenched chiral condensate. This may appear surprising, since the chiral condensate is just determined from the partition function through, for example, a formula like (1.5). If the de Wit-'t Hooft poles cancel in the partition function, how can they not cancel after taking the $\mu_{v}$-derivative and the limit $N_{v} \rightarrow 0$ as in eq. (1.5)? The answer is simple. To be able to take the quenched limit of $N_{v} \rightarrow 0$ we must not cross any singularities that can obstruct the analytical continuation. This is, however, precisely what happens for those terms in the expansion (3.31) that, for given $N$, are singular. To give an example, let us consider the $\mu_{v}^{3}$-term in the expansion (3.31). For simplicity, let us also consider the case where all physical masses vanish. Then there is only one term:

$$
\frac{\mu_{v}^{3}}{8 N\left(N^{2}-1\right)} \text {. }
$$

This term is singular for $N=1$ (and, of course, also in the more trivial case of $N=0$ ), and therefore cannot possibly represent the partially quenched chiral condensate to order $\mu_{v}^{3}$ in the $N=1$ theory. Let us therefore trace what happens if we set $N_{f}+\nu=1$ from the outset. The only term in the expansion (2.13) that gives an $\mathcal{O}\left(\mu_{v}^{3}\right)$ contribution to $\Sigma_{\nu}\left(\mu_{v}\right)$ in this massless case is $\left(N \equiv N_{f}+N_{v}+\nu\right)$

$$
\begin{aligned}
\frac{1}{32 N\left(N^{2}-1\right)} \operatorname{Tr}\left(\mathcal{M}^{\dagger} \mathcal{M}\right)^{2} & =\frac{N_{v} \mu_{v}^{4}}{32 N\left(N^{2}-1\right)} \\
& =\frac{\mu_{v}^{4}}{32\left(N_{v}+1\right)\left(N_{v}+2\right)}
\end{aligned}
$$

which precisely in this $N_{f}+\nu=1$ case is not linear in $N_{v}$. The factor $1 / N_{v}$ in the expression for the quenched condensate (3.30) will in this case remain uncancelled, and an analytic continuation to $N_{v}=0$ is prohibited by the singularity. The term (3.32) in the expansion (3.31) is therefore incorrect precisely in the case $N_{f}+\nu=1$ (but it is valid for all $N_{f}+\nu>1$ ). This holds also for all higher orders in the expansion. The small-mass expansion for $\Sigma_{\nu}$ is thus given to a finite number of terms. For example, in the massless $N_{f}+\nu=3$ theory we obtain

$$
\frac{\Sigma_{\nu}\left(\mu_{v},\{\mu\}\right)}{\Sigma}=\frac{\nu}{\mu_{v}}+\frac{\mu_{v}}{6}-\frac{\mu_{v}^{3}}{192}+\frac{\mu_{v}^{5}}{1920}+\ldots, \quad N_{f}+\nu=3
$$


where the higher-order terms in $\mu_{v}$ cannot be purely power-like. The same phenomenon occurs for larger integer values of $N$, where we encounter the singularity at higher orders. We thus conclude that the small-mass expansion (3.31) is valid up to the first term where a pole appears. The obstruction to an all-order expansion of $\Sigma_{\nu}\left(\mu_{v},\left\{\mu_{i}\right\}\right)$ is from this point of view uncancelled de Wit-'t Hooft poles. The expansion can of course be pushed to very high orders by considering $N_{f}+\nu$ sufficiently large. We stress again that the expansion is perfectly meaningful up to the order at which the first uncancelled de Wit-'t Hooft pole appears, and that it is completely understood why the expansion cannot be pushed beyond this order. It is not a peculiar and unwanted artifact of the replica method, but a real feature of the small-mass expansion of the partially quenched chiral condensate.

With this caveat in mind, we can now check the expansion (3.31) against both the supersymmetric method and results from Random Matrix Theory. For instance, both methods yield a fully quenched chiral condensate of the form [10, , ]

$$
\frac{\Sigma_{\nu}\left(\mu_{v}\right)}{\Sigma}=\mu_{v}\left[I_{\nu}\left(\mu_{v}\right) K_{\nu}\left(\mu_{v}\right)+I_{\nu+1}\left(\mu_{v}\right) K_{\nu-1}\left(\mu_{v}\right)\right]+\frac{\nu}{\mu_{v}} .
$$

where $I_{n}(x)$ and $K_{n}(x)$ are modified Bessel functions. Looking at the small- $\mu_{v}$ expansions of $K_{n}\left(\mu_{v}\right)$, one notices that they are only purely power-like up to order $n-2$ (after which a logarithmic term must be included). The small-mass expansion of the closed expression (3.35) therefore cannot possibly match to all orders the purely power-like expansion that would seem to follow from the small-mass expansion of the partition function in eq. (2.13). And we find that the agreement of the two expansions is exact precisely up to the order at which our small-expansion (3.31) ceases to be valid. The appearance, at higher orders, of logarithmic terms in the expansion explicitly confirms the statement above: there is simply no power-law small-mass expansion of the chiral condensate beyond that given order. It is quite remarkable that the normally cancelling de Wit-'t Hooft poles precisely obstruct the small-mass expansion at just the right order.

We have also checked that the double-expansions in both $\mu_{v}$ and physical fermion masses $\mu_{i}$ are correct precisely up to the order at which eq. (3.31) is valid. We have done this by comparing the small-mass expansions of the partially quenched chiral condensate in the $N_{f}=1$ theory with the expansions of the closed expression

$$
\begin{aligned}
\frac{\Sigma_{\nu}\left(\mu_{v}, \mu\right)}{\Sigma}= & \mu_{v}\left[I_{\nu+1}\left(\mu_{v}\right) K_{\nu+1}\left(\mu_{v}\right)+I_{\nu+2}\left(\mu_{v}\right) K_{\nu}\left(\mu_{v}\right)\right]+\frac{\nu}{\mu_{v}} \\
& +2 \mu \frac{K_{\nu}\left(\mu_{v}\right)}{I_{\nu}(\mu)} \frac{\mu_{v} I_{\nu}\left(\mu_{v}\right) I_{\nu+1}(\mu)-\mu I_{\nu}(\mu) I_{\nu+1}\left(\mu_{v}\right)}{\mu_{v}^{2}-\mu^{2}},
\end{aligned}
$$

which has been derived by the supersymmetric method [7] (and which can also easily been seen to agree with the Random Matrix Theory result). Again we find perfect agreement with all terms up to the order at which our expansion (3.31) ceases to be valid, i.e. up to the first uncancelled de Wit-'t Hooft pole.

\subsection{The large-mass expansion}

Having succeeded in deriving the low-mass expansion for the partially quenched chiral condensate, we next turn to the opposite expansion, i.e. for large masses. The advantage of this expansion is that it can be pushed to arbitrarily high order, for any value of $N_{f}$.

Recall that the effective partition function (2.1) only depends on the combination $\mathcal{M}^{\dagger} \mathcal{M}$ for $\nu=0$.

This combination is hermitian and there are thus only $N_{f}$ degrees of freedom in $\mathcal{Z}_{0}^{\left(N_{f}\right)}$, represented 
for instance by the eigenvalues $\mu_{i}^{2}$ of $\mathcal{M}^{\dagger} \mathcal{M}$. In terms of $\mu_{i}$ the expansion variables of eq. (2.6) read

$$
t_{k}^{-} \equiv-\frac{2^{2 k+1}}{2 k+1} \sum_{i=1}^{N_{f}} \mu_{i}^{-(2 k+1)} .
$$

The idea is now to find suitable partition function constraints that will enable us to solve for the partition function in an expansion in the variables $t_{k}^{-}$. This is not completely straightforward, but as shown first by Gross and Newman [13], it is possible to recover a complete set of Virasoro constraints in the variables $t_{k}^{-}$if one first extracts a simple prefactor from the partition function. The factorization is as follows [13] (see also ref. [14] for the generalization to other types of matrix integrals):

$$
\mathcal{Z}_{0}^{\left(N_{f}\right)}=\left(\prod_{a, b}^{N_{f}}\left(\frac{1}{2} \mu_{a}+\frac{1}{2} \mu_{b}\right)^{-1 / 2}\right) e^{\sum_{b} \mu_{b}} Y_{0}^{\left(N_{f}\right)}\left(\left\{t_{k}^{-}\right\}\right) .
$$

The exponential prefactor indicates that an expansion of the partition function entirely in terms of the $t_{k}^{-}$'s simply is not possible. This is also obvious if we consider the original group integral in a saddlepoint approximation around large $\mathcal{M}$ : the leading behaviour is indeed exponentially large. Moreover, one suspects from the form $(3.38)$ which is essentially a saddle-point expansion that the resulting series expansion we will derive below is at best asymptotic. This is in contrast to the previously considered small-mass expansion, which we believe is convergent.

The remaining factor $Y_{0}^{\left(N_{f}\right)}\left(\left\{t_{k}^{-}\right\}\right)$in eq. (3.38) turns out to be annihilated by an infinite set of Virasoro operators. Explicitly, by defining

$$
\begin{aligned}
& \mathcal{L}_{0}^{-} \equiv \sum_{k=0}^{\infty}\left(k+\frac{1}{2}\right) t_{k}^{-} \frac{\partial}{\partial t_{k}^{-}}+\frac{1}{16}+\frac{\partial}{\partial t_{0}^{-}}, \\
& \mathcal{L}_{n}^{-} \equiv \sum_{k=0}^{\infty}\left(k+\frac{1}{2}\right) t_{k}^{-} \frac{\partial}{\partial t_{k+n}^{-}}+\frac{1}{4} \sum_{k=1}^{n} \frac{\partial^{2}}{\partial t_{k-1}^{-} \partial t_{n-k}^{-}}+\frac{\partial}{\partial t_{n}^{-}}, \quad n \geq 1
\end{aligned}
$$

the function $Y_{0}^{\left(N_{f}\right)}\left(\left\{t_{k}^{-}\right\}\right)$of $(3.38)$ is found to satisfy 13

$$
\mathcal{L}_{n}^{-} Y_{0}^{\left(N_{f}\right)}=0 \quad n \geq 0
$$

One readily verifies that these constraints indeed also fulfill the commutation relations of classical Virasoro generators (2.9), and thus form a consistent set of constraints. These constraints are also complete in that they determine the partition function uniquely, given the boundary condition $Y_{0}^{\left(N_{f}\right)}=$ 1 for $t_{k}^{-}=0$, i.e. in the limit of infinite masses. While these constraint equations were already established in ref. [13], they have not previously been used to derive a systematic expansion for the partition function. We do this by copying the procedure of the small-mass expansion. That is, we expand the unknown function $Y_{0}^{\left(N_{f}\right)}\left(\left\{t_{k}^{-}\right\}\right)$as follows:

$$
Y_{0}^{\left(N_{f}\right)}\left(\left\{t_{k}^{-}\right\}\right) \equiv 1+\sum_{k=0}^{\infty} c_{k} t_{k}^{-}+\sum_{0 \leq k_{1} \leq k_{2}} c_{k_{1}, k_{2}} t_{k 1}^{-} t_{k_{2}}^{-}+\ldots
$$

We next solve for the coefficients $c_{k_{1}, \ldots, k_{n}}$ iteratively. In fact, this is in many ways simpler than in the small-mass expansion in that we in this case easily can derive closed analytical expressions to all 
orders. For example, we find the following simple formula for the first strings of coefficients:

$$
\underset{\substack{0,0, \ldots, 0, k, \ldots, k \\ n+1}}{c_{m}}=\left(\prod_{j=1}^{n} \frac{-1}{j+1} \frac{8 m(2 k+1)+8 j+1}{16}\right) c_{\substack{0, k, \ldots, k, m}} .
$$

Using this formula, and others similar, it is straightforward to derive very high order expansions for $Y_{0}^{\left(N_{f}\right)}\left(\left\{t_{k}^{-}\right\}\right)$, and therefore also for the partition function itself. For example, to $7^{\text {th }}$ order in the masses we find a rather formidable-looking expression (that is easily pushed to much higher orders):

$$
\begin{aligned}
\mathcal{Z}_{0}^{\left(N_{f}\right)}= & \left(\prod_{a, b}^{N_{f}}\left(\frac{1}{2} \mu_{a}+\frac{1}{2} \mu_{b}\right)^{-1 / 2}\right) \exp \left[\sum_{b} \mu_{b}\right] \\
& {\left[1+\frac{1}{8} \operatorname{Tr}\left(\mathcal{M}^{\dagger} \mathcal{M}\right)^{-1 / 2}+\frac{9}{128}\left(\operatorname{Tr}\left(\mathcal{M}^{\dagger} \mathcal{M}\right)^{-1 / 2}\right)^{2}\right.} \\
& +\frac{51}{1024}\left(\operatorname{Tr}\left(\mathcal{M}^{\dagger} \mathcal{M}\right)^{-1 / 2}\right)^{3}+\frac{3}{128} \operatorname{Tr}\left(\mathcal{M}^{\dagger} \mathcal{M}\right)^{-3 / 2}+\frac{1275}{32768}\left(\operatorname{Tr}\left(\mathcal{M}^{\dagger} \mathcal{M}\right)^{-1 / 2}\right)^{4} \\
& +\frac{75}{1024}\left(\operatorname{Tr}\left(\mathcal{M}^{\dagger} \mathcal{M}\right)^{-1 / 2}\right)\left(\operatorname{Tr}\left(\mathcal{M}^{\dagger} \mathcal{M}\right)^{-3 / 2}\right)+\frac{8415}{262144}\left(\operatorname{Tr}\left(\mathcal{M}^{\dagger} \mathcal{M}\right)^{-1 / 2}\right)^{5} \\
& +\frac{2475}{16384}\left(\operatorname{Tr}\left(\mathcal{M}^{\dagger} \mathcal{M}\right)^{-1 / 2}\right)^{2}\left(\operatorname{Tr}\left(\mathcal{M}^{\dagger} \mathcal{M}\right)^{-3 / 2}\right)+\frac{45}{1024} \operatorname{Tr}\left(\mathcal{M}^{\dagger} \mathcal{M}\right)^{-5 / 2}+\frac{115005}{4194304}\left(\operatorname{Tr}\left(\mathcal{M}^{\dagger} \mathcal{M}\right)^{-1 / 2}\right)^{6} \\
& +\frac{33825}{131072}\left(\operatorname{Tr}\left(\mathcal{M}^{\dagger} \mathcal{M}\right)^{-1 / 2}\right)^{3}\left(\operatorname{Tr}\left(\mathcal{M}^{\dagger} \mathcal{M}\right)^{-3 / 2}\right)+\frac{6075}{98304}\left(\operatorname{Tr}\left(\mathcal{M}^{\dagger} \mathcal{M}\right)^{-3 / 2}\right)^{2} \\
& +\frac{1845}{8192}\left(\operatorname{Tr}\left(\mathcal{M}^{\dagger} \mathcal{M}\right)^{-1 / 2}\right)\left(\operatorname{Tr}\left(\mathcal{M}^{\dagger} \mathcal{M}^{-5 / 2}\right)+\frac{805035}{33554432}\left(\operatorname{Tr}\left(\mathcal{M}^{\dagger} \mathcal{M}\right)^{-1 / 2}\right)^{7}\right. \\
& +\frac{1657425}{4194304}\left(\operatorname{Tr}\left(\mathcal{M}^{\dagger} \mathcal{M}\right)^{-1 / 2}\right)^{4}\left(\operatorname{Tr}\left(\mathcal{M}^{\dagger} \mathcal{M}\right)^{-3 / 2}\right)+\frac{99225}{262144}\left(\operatorname{Tr}\left(\mathcal{M}^{\dagger} \mathcal{M}\right)^{-1 / 2}\right)\left(\operatorname{Tr}\left(\mathcal{M}^{\dagger} \mathcal{M}\right)^{-3 / 2}\right)^{2} \\
& \left.+\frac{90405}{131072}\left(\operatorname{Tr}\left(\mathcal{M}^{\dagger} \mathcal{M}\right)^{-1 / 2}\right)^{2}\left(\operatorname{Tr}\left(\mathcal{M}^{\dagger} \mathcal{M}\right)^{-5 / 2}\right)+\frac{7875}{32768} \operatorname{Tr}\left(\mathcal{M}^{\dagger} \mathcal{M}\right)^{-7 / 2}+\ldots\right]
\end{aligned}
$$

In writing (2.6) and (3.37) we have implicitly assumed that $\mathcal{M}^{\dagger} \mathcal{M}$ is invertible. This means that there must be no zero-eigenvalues in the mass matrix, and we thus by default cannot consider any number of massless fermions in this large-mass expansion. In particular, the concept of flavor-topology duality, by which a topological charge $\nu$ is implemented by adding $\nu$ massless fermions to the $\nu=0$ partition function, cannot be applied in the framework of a large-mass expansion. Because the Virasoro constraints (3.39) and (3.40) apply only to the case $\nu=0$ we know of no analogous way to implement this large-mass expansion outside of the $\nu=0$ sector.

Introducing $N_{v}$ mass-degenerate valence quarks into the large-mass expansion of the effective partition function, and noting that in this case

$$
\operatorname{Tr}\left(\mathcal{M}^{\dagger} \mathcal{M}\right)^{-1 / 2}=\operatorname{Tr}(M)^{-1}+N_{v} \mu^{-1}
$$

we again observe that $N_{v}$ can be regarded as a continuous parameter. The replica approach can therefore again be applied.

Using the definition (3.30) and a good handful of simple algebra we find the partially quenched chiral condensate for large masses:

$$
\frac{\Sigma_{0}\left(\mu_{v},\{\mu\}\right)}{\Sigma}=1-\sum_{i=1}^{N_{f}} \frac{1}{\mu_{i}+\mu_{v}}
$$




$$
\begin{aligned}
& -\frac{1}{\mu_{v}^{2}}\left[\frac{1}{8}+\frac{1}{8} \operatorname{Tr}\left(\frac{1}{M}\right)+\frac{1}{8}\left(\operatorname{Tr}\left(\frac{1}{M}\right)\right)^{2}+\frac{9}{128} \operatorname{Tr}\left(\frac{1}{M^{3}}\right)\right. \\
& +\frac{1}{8}\left(\operatorname{Tr}\left(\frac{1}{M}\right)\right)^{3}+\frac{1}{8}\left(\operatorname{Tr}\left(\frac{1}{M}\right)\right)^{4}+\frac{9}{32} \operatorname{Tr}\left(\frac{1}{M^{3}}\right) \operatorname{Tr}\left(\frac{1}{M}\right)+\frac{1}{8}\left(\operatorname{Tr}\left(\frac{1}{M}\right)\right)^{5} \\
& \left.+\frac{45}{64} \operatorname{Tr}\left(\frac{1}{M^{3}}\right)\left(\operatorname{Tr}\left(\frac{1}{M}\right)\right)^{2}+\frac{225}{1024} \operatorname{Tr}\left(\frac{1}{M^{5}}\right)+\ldots\right] \\
& -\frac{1}{\mu_{v}^{4}}\left[\frac{9}{128}+\frac{27}{128} \operatorname{Tr}\left(\frac{1}{M}\right)+\frac{27}{64}\left(\operatorname{Tr}\left(\frac{1}{M}\right)\right)^{2}+\frac{45}{64}\left(\operatorname{Tr}\left(\frac{1}{M}\right)\right)^{3}+\frac{189}{512} \operatorname{Tr}\left(\frac{1}{M^{3}}\right)+\ldots\right] \\
& -\frac{1}{\mu_{v}^{6}}\left[\frac{225}{1024}+\frac{1125}{1024} \operatorname{Tr}\left(\frac{1}{M}\right)+\ldots\right]
\end{aligned}
$$

This expansion agrees with the asymptotic expansion of the analytical expressions for $\Sigma\left(\mu_{v},\{\mu\}\right)$ found in [7] for $N_{f}=0$ and $N_{f}=1$.

\section{Massive spectral sum rules}

The spectral sum rules presented in section 1 concern inverse moments of the Dirac eigenvalues averaged with respect to the massless theory. These massless spectral sum rules are, however, only special cases of massive spectral sum rules obtained by averaging with respect to the massive theory. In fact, massive spectral sum rules appear quite naturally in lattice simulations. Such massive spectral sum rules are conventionally taken [3, 19] to be of the form

$$
\int_{0}^{\infty} d \zeta \rho_{S}\left(\zeta ;\left\{\mu_{i}\right\}\right) \prod_{j} \frac{1}{\left(\zeta^{2}+\mu_{j}^{2}\right)^{n_{j}}},
$$

for given integer $n_{j}$ 's. Here masses and Dirac operator eigenvalues are treated on equal footing in the denominators. However, we could equally well define massive spectral sum rules by

$$
\left\langle\sum_{\zeta_{j}>0} \frac{1}{\zeta_{j}^{2 n}}\right\rangle=\int_{0}^{\infty} d \zeta \frac{\rho_{S}\left(\zeta ;\left\{\mu_{i}\right\}\right)}{\zeta^{2 n}},
$$

which are just the usual inverse moments, but evaluated in the massive theory. Here and below the brackets denote the average with respect to the massive and partially quenched theory in a topological gauge field sector of charge $\nu$. In order to derive such massive spectral sum rules we return to the small-mass expansion of the partially quenched condensate (3.31).

Inserting the original partition function (1.1) into the definition (3.30) of the partially quenched chiral condensate one has

$$
\frac{\Sigma_{\nu}\left(\mu_{v},\{\mu\}\right)}{\Sigma}=2 \mu_{v}\left\langle\sum_{\zeta_{n}>0} \frac{1}{\zeta_{n}^{2}+\mu_{v}^{2}}\right\rangle+\frac{\nu}{\mu_{v}} .
$$

We can now find a useful general relation between the partially quenched chiral condensate, and (massive) spectral sum rules. The connection is simple: the sum rules of inverse moments $\zeta^{-2 n}$ of the eigenvalues will be convergent up to a given value of $n$, which we denote by $k$. Let us therefore expand the denominator of eq. (4.48) in partial fractions up to this maximal value $k$ :

$$
\frac{1}{\zeta_{n}^{2}+\mu_{v}^{2}}=\frac{1}{\zeta_{n}^{2}}-\frac{\mu_{v}^{2}}{\zeta_{n}^{4}}+\ldots+\frac{(-1)^{k} \mu_{v}^{2 k}}{\zeta_{n}^{2 k}\left(\zeta_{n}^{2}+\mu_{v}^{2}\right)}
$$


which means that

$$
\frac{\Sigma_{\nu}\left(\mu_{v},\{\mu\}\right)}{\Sigma}=2\left\langle\sum_{\zeta_{n}>0} \frac{1}{\zeta_{n}^{2}}\right\rangle \mu_{v}-2\left\langle\sum_{\zeta_{n}>0} \frac{1}{\zeta_{n}^{4}}\right\rangle \mu_{v}^{3}+\ldots+\left\langle\sum_{\zeta_{n}>0} \frac{2(-1)^{k} \mu_{v}^{2 k+1}}{\zeta_{n}^{2 k}\left(\zeta_{n}^{2}+\mu_{v}^{2}\right)}\right\rangle+\frac{\nu}{\mu_{v}} .
$$

We can thus simply read off a whole string of massive spectral sum rules from the coefficients in the $\mu_{v}$-expansion of the partially quenched chiral condensate (3.31):

$$
\begin{aligned}
& \left\langle\sum_{\zeta_{n}>0} \frac{1}{\zeta_{n}^{2}}\right\rangle=\frac{1}{4 N}+\frac{\operatorname{Tr} M^{2}}{16 N^{2}\left(N^{2}-1\right)}+\frac{\left(\operatorname{Tr} M^{2}\right)^{2}}{16 N^{3}\left(N^{2}-1\right)\left(N^{2}-4\right)} \\
& -\frac{\operatorname{Tr}\left(\left(M^{2}\right)^{2}\right)}{32 N^{2}\left(N^{2}-1\right)\left(N^{2}-4\right)}+\frac{15 \operatorname{Tr}\left(\left(M^{2}\right)^{3}\right)}{768 N^{2}\left(N^{2}-1\right)\left(N^{2}-4\right)\left(N^{2}-9\right)} \\
& +\frac{3\left(2 N^{2}-3\right)\left(\operatorname{Tr} M^{2}\right)^{3}}{64 N^{4}\left(N^{2}-1\right)^{2}\left(N^{2}-4\right)\left(N^{2}-9\right)} \\
& +\frac{3\left(3-2 N^{2}\right) \operatorname{Tr} M^{2} \operatorname{Tr}\left(\left(M^{2}\right)^{2}\right)}{64 N^{3}\left(N^{2}-1\right)^{2}\left(N^{2}-4\right)\left(N^{2}-9\right)}+\ldots \\
& \left\langle\sum_{\zeta_{n}>0} \frac{1}{\zeta_{n}^{4}}\right\rangle=\frac{1}{16 N\left(N^{2}-1\right)}+\frac{\operatorname{Tr} M^{2}}{16 N^{2}\left(N^{2}-1\right)\left(N^{2}-4\right)} \\
& +\frac{\left(6 N^{2}-9\right)\left(\operatorname{Tr} M^{2}\right)^{2}}{64 N^{3}\left(N^{2}-1\right)^{2}\left(N^{2}-4\right)\left(N^{2}-9\right)} \\
& -\frac{3\left(3 N^{2}-7\right) \operatorname{Tr}\left(\left(M^{2}\right)^{2}\right)}{256 N^{2}\left(N^{2}-1\right)^{2}\left(N^{2}-4\right)\left(N^{2}-9\right)}+\ldots \\
& \left\langle\sum_{\zeta_{n}>0} \frac{1}{\zeta_{n}^{6}}\right\rangle=\frac{1}{32 N\left(N^{2}-1\right)\left(N^{2}-4\right)}+\frac{15 \operatorname{Tr} M^{2}}{256 N^{2}\left(N^{2}-1\right)\left(N^{2}-4\right)\left(N^{2}-9\right)}+\ldots \\
& \left\langle\sum_{\zeta_{n}>0} \frac{1}{\zeta_{n}^{8}}\right\rangle=\frac{5}{256 N\left(N^{2}-1\right)\left(N^{2}-4\right)\left(N^{2}-9\right)}+\ldots
\end{aligned}
$$

The resulting sum rules are here only given as a perturbative expansion in the physical fermion masses, in contrast to the massless spectral sum rules which are exact. One notices that trivially the "diagonal" massless spectral sum rules (2.14)-(2.17) are reproduced by taking all $\mu_{i}=0$. As discussed already in section 2 , the above expansions are valid for $N$ larger than the biggest integer poles. This is completely understandable from the present point of view, as it corresponds to performing the expansion (4.49) in partial fractions only up to the point where the spectral sums of all terms still converge. The "non-diagonal" massive sum rules can also be calculated within this framework. One simply needs to break the mass-degeneracy of the valence quarks and consider general partially quenched $n$-point correlators of $\bar{\psi} \psi$, as discussed in the introduction.

These new massive spectral sum rules can easily be compared with the predictions from Random Matrix Theory. What we need is to expand the microscopic spectral density $\rho_{S}^{\left(N_{f}, \nu\right)}\left(\zeta,\left\{\mu_{i}\right\}\right)$ in powers of $\operatorname{Tr} M^{2}$, and then insert this expansion in

$$
\frac{\Sigma_{\nu}\left(\mu_{v},\left\{\mu_{i}\right\}\right)}{\Sigma}=2 \mu_{v} \int_{0}^{\infty} d \zeta \frac{\rho_{S}^{\left(N_{f}, \nu\right)}(\zeta,\{\mu\})}{\zeta^{2}+\mu_{v}^{2}}+\frac{\nu}{\mu_{v}} .
$$

For example, for $N_{f}=1$ the microscopic spectral density for a massive fermion in a gauge field sector 
of arbitrary topological index $\nu$ is given by [19] (see also the second reference of [5] and ref. [20]):

$$
\begin{aligned}
\rho_{S}^{(\nu)}(\zeta ; \mu)= & \frac{|\zeta|}{2}\left[J_{\nu+1}(\zeta)^{2}-J_{\nu}(\zeta) J_{\nu+2}(\zeta)\right]+\frac{|\zeta| \mu^{2} J_{\nu}(\zeta)}{2(\nu+1)\left(\zeta^{2}+\mu^{2}\right)}\left[\frac{I_{\nu+2}(\mu)}{I_{\nu}(\mu)} J_{\nu}(\zeta)+J_{\nu+2}(\zeta)\right] \\
= & \frac{|\zeta|}{2}\left[J_{\nu+1}(\zeta)^{2}-J_{\nu}(\zeta) J_{\nu+2}(\zeta)\right]+\frac{J_{\nu}(\zeta) J_{\nu+2}(\zeta)}{2(\nu+1)|\zeta|} \mu^{2} \\
& +\frac{1}{2(\nu+1)}\left(\frac{J_{\nu}(\zeta)}{4(\nu+1)(\nu+2)|\zeta|}-\frac{J_{\nu+2}(\zeta)}{|\zeta|^{3}}\right) J_{\nu}(\zeta) \mu^{4}+\ldots
\end{aligned}
$$

Inserting this expansion into (4.55), we find that the relevant integrals become analytically doable when $\nu$ is taken large enough to make the integrals converge. In this way we have explicitly confirmed a number of terms in the above expansions of the massive spectral sum rules.

\section{Generalizations and outlook}

The small and large mass expansions for the partially quenched chiral condensate can of course in principle be extended to the two other major universality classes, corresponding to $\mathrm{SU}(2)$ gauge group with fermions in the fundamental representation, and $\mathrm{SU}\left(N_{c} \geq 2\right)$ with fermions in the adjoint representation. The challenge is here to find a convenient method that permits high-order expansions. In our present case all simplifications arose from the observation that the effective partition function is annihilated by two sets of Virasoro constraints, which in turn is rooted in the fact that this effective partition function is a $\tau$-function of an integrable KP hierarchy. The effective partition functions of the two other universality classes have not been nearly as well studied, although there are reasons to believe that they are what is known as "Pfaffian $\tau$-functions" (see, e.g., ref. [21]). It may thus be possible to derive analogous partition function constraints, which will permit high-order expansions with little labor. In this connection we also make the following observations. The Virasoro constraints (2.8) and (3.41) both follow from the simple differential equation

$$
\frac{\partial^{2} \mathcal{Z}_{0}^{\left(N_{f}\right)}}{\partial \mathcal{M}_{i k} \partial \mathcal{M}_{k j}^{\dagger}}=\delta_{i j} \frac{1}{4} \mathcal{Z}_{0}^{\left(N_{f}\right)}
$$

by changing variables to the pertinent expansion parameters. This differential equation by itself is a trivial consequence of the fact that the integration manifold is $\mathrm{U}\left(N_{f}+N_{v}\right)$, as it just amounts to inserting the unitarity condition $U^{\dagger} U=1$ in the integrand of the effective partition function (2.1). Imposing two boundary conditions is thus sufficient to determine the partition function uniquely. In the case at hand it is the choice $\mathcal{Z}_{0}^{\left(N_{f}\right)}\left(t_{k}^{+}=0\right)=1$ and $Y_{0}^{\left(N_{f}\right)}\left(t_{k}^{-}=0\right)=1$, together with the required property $\mathcal{Z}_{0}^{\left(N_{f}\right)}\left(\mathcal{M}, \mathcal{M}^{\dagger}\right)=\mathcal{Z}_{0}^{\left(N_{f}\right)}\left(\mathcal{M}^{\dagger} \mathcal{M}\right)$ that provide the two conditions.

Except for a change of the proportionality constant, here 1/4, the differential equation (5.1) also holds for the effective partition functions of the two other universality classes mentioned above, as well as for the one of $\mathrm{QCD}_{3}$. It is also instructive to notice that the restriction to a sector of fixed topological charge does not affect the differential equation. The boundary conditions imposed on the differential equation, however, separate the solutions, i.e., the partition functions. For instance, before the projection onto a fixed topological sector the partition function studied here $\operatorname{depends}$ on $\operatorname{det}(\mathcal{M})$ and $\operatorname{det}\left(\mathcal{M}^{\dagger}\right)$ as well as on $\mathcal{M} \mathcal{M}^{\dagger}$.

Of course we can already at this stage obtain the partially quenched chiral condensates to lowest nontrivial orders for the two remaining ensembles by simply making use of the same small-mass expansions 
of the partition function that were used to derive the first sum rules. This is a trivial exercise: from ref. [2] we learn that the effective partition function for $\mathrm{SU}(2)$ gauge group and $N_{f}+N_{v}$ fermions in the fundamental representation is

$$
\mathcal{Z}_{\nu}=[\operatorname{Pf}(\tilde{\mathcal{M}})]^{\nu}\left(1+\frac{1}{8\left(2 N_{f}+2 N_{v}+\nu-1\right)} \operatorname{Tr} \tilde{\mathcal{M}}^{\dagger} \tilde{\mathcal{M}}+\ldots\right),
$$

where, now $\tilde{\mathcal{M}}$ is an antisymmetric $\left(2 N_{f}+2 N_{v}\right) \times\left(2 N_{f}+2 N_{v}\right)$ matrix with the usual mass matrices $+\mathcal{M}$ and $-\mathcal{M}$ placed in the two off-diagonal blocks, and zeros in the two diagonal blocks. Using again eqs. (3.29) and (3.30) we find the partially quenched chiral condensate to this order:

$$
\frac{\Sigma_{\nu}\left(\mu_{v}\right)}{\Sigma}=\frac{\nu}{\mu_{v}}+\frac{\mu_{v}}{2\left(2 N_{f}+\nu-1\right)}+\ldots
$$

which one can confirm matches the first term in the expansion of the result obtained from Random Matrix Theory (see the first of ref. [11]). Note that this expansion has its first uncancelled de Wit-'t Hooft pole at $2 N_{f}+\nu=1$, and the above term in the expansion is thus valid for $2 N_{f}+\nu>1$.

Similarly, for the universality class corresponding to $N_{f}+N_{v}$ adjoint fermions and arbitrary gauge group $\mathrm{SU}\left(N_{c}\right)$ we see from ref. [2] that the partition function expansion is

$$
\mathcal{Z}_{\bar{\nu}}=\operatorname{det}(\mathcal{M})^{\bar{\nu}}\left(1+\frac{1}{2\left(N_{f}+N_{v}+2 \bar{\nu}+1\right)} \operatorname{Tr} \mathcal{M}^{\dagger} \mathcal{M}+\ldots\right),
$$

where $\bar{\nu}=\nu N_{c}$ is an integer. Using the present replica method this leads to a partially quenched chiral condensate of

$$
\frac{\Sigma_{\bar{\nu}}\left(\mu_{v}\right)}{\Sigma}=\frac{\bar{\nu}}{\mu_{v}}+\frac{\mu_{v}}{N_{f}+2 \bar{\nu}+1}+\ldots,
$$

which one again can check matches the result from Random Matrix Theory [11]. There is no de Wit-'t Hooft pole in this case, and thus no retriction on the validity of this first term.

Since one can obtain perturbative solutions to the partially quenched chiral condensate in all three universality classes, a natural question concerns the microscopic spectral density of the Dirac operator $\rho_{S}\left(\zeta ;\left\{\mu_{i}\right\}\right)$. This density is given by the discontinuity of the partially quenched chiral condensate across the cut on the imaginary axis [7]:

$$
\rho_{S}\left(\zeta ;\left\{\mu_{i}\right\}\right)=\frac{1}{2 \pi} \operatorname{Disc}_{\mu_{v}=i \zeta} \Sigma_{\nu}\left(\mu_{v},\left\{\mu_{i}\right\}\right)=\frac{1}{2 \pi} \lim _{\epsilon \rightarrow 0}\left[\Sigma_{\nu}\left(i \zeta+\epsilon,\left\{\mu_{i}\right\}\right)-\Sigma_{\nu}\left(i \zeta-\epsilon,\left\{\mu_{i}\right\}\right)\right] .
$$

(This identification holds when one considers $\Sigma_{\nu}\left(\mu_{v},\left\{\mu_{i}\right\}\right)$ as a function of a real mass $\mu_{v}$, and then replaces $\left.\mu_{v} \rightarrow i \zeta \pm \epsilon\right)$. It now seems straightforward to insert our small-mass and large-mass series expansions for $\Sigma_{\nu}\left(\mu_{v},\left\{\mu_{i}\right\}\right)$, and derive corresponding series expansions for the spectral density $\rho_{S}\left(\zeta ;\left\{\mu_{i}\right\}\right)$. But this is not possible. Let us first consider the small-mass expansion of eq. (3.31). As we emphasized in section 3.1, this expansion is valid up to the first de Wit-'t Hooft pole. Except for the topological term $\nu / \mu_{v}$ which always trivially yields the correct $\delta$-function contribution to the microscopic spectral density, this power-series expansion of a finite number of terms has no cut across the imaginary axis. This may seem surprising, since the microscopic spectral density $\rho_{S}\left(\zeta ;\left\{\mu_{i}\right\}\right)$ does have a simple and well-defined power-series expansion. The explanation is, however, simple. Because of the de Wit-'t Hooft poles, we can derive the small-mass expansion of $\Sigma_{\nu}\left(\mu_{v},\left\{\mu_{i}\right\}\right)$ up to (and including) order $\mu_{v}^{2 N_{f}+2 \nu-1}$. But the perturbatively expanded $\rho_{S}\left(\zeta ;\left\{\mu_{i}\right\}\right)$ (see, e.g. the massless case 
(2.27)) starts only at order $\zeta^{2 N_{f}+2 \nu+1}$. The microscopic spectral density is always precisely one step ahead of the order to which we can push the small-mass expansion of the partially quenched chiral condensate! In hindsight, a phenomenon like this had to occur, since the pure power series implied by the small-mass expansion cannot give rise to any discontinuity across the imaginary axis. So the de Wit-'t Hooft poles in fact precisely save what would otherwise be a paradoxical situation.

The asymptotic large-mass expansion of section 3.2 cannot be used to extract the microscopic spectral density either. Here the reason is very different. Recall that the asymptotic expansion we have derived in section 3.2 is suitable $\mu_{v}$ on the positive real axis. For example, for $N_{f}=1$ our expansion simply coincides, up to an irrelevant normalization factor, with the asymptotic expansion of the Bessel function $I_{0}(\mu)$ :

$$
\mathcal{Z}_{0}^{\left(N_{f}=1\right)}=I_{0}(\mu) \sim \frac{e^{\mu}}{\sqrt{2 \pi \mu}} \sum_{k=0}^{\infty} \frac{(-1)^{k}}{(2 \mu)^{k}} \frac{\Gamma(k+1 / 2)}{k ! \Gamma(-k+1 / 2)}
$$

This asymptotic expansion is correct, but it neglects exponentially suppressed terms of order $e^{-\mu} / \sqrt{\mu}$ and lower. Because of this, the asymptotic expansion (5.7) does not reproduce the asymptotic expansion for $J_{0}(\mu)$ after rotation to the imaginary axis (the exponentially suppressed terms then become of magnitude comparable to those kept in (5.7), and that expansion is therefore no longer correct near the imaginary axis). We conclude that neither of the two expansions we have considered here are suitable for deriving the microscopic spectral density.

\section{Conclusions}

Using the Virasoro constraints on the effective partition function we have obtained small-mass and large-mass expansions for the QCD partition function valid in the scaling region $V \ll 1 / m_{\pi}^{4}$. In these expansions we can treat the number of valence quarks $N_{v}$ as a continuous parameter. This form is thus suited for a calculation of the partially quenched chiral condensate using the replica method. Two series of spectral sum rules for the QCD Dirac operator follow from the small-mass expansion. One series extends the Leutwyler-Smilga sum rules in a simple way to very high (here $8^{\text {th }}$ ) order, while the other series is new: it computes the spectral sum rules in the massive theory, and we have given series expansions in the physical masses for these new sum rules. In all cases we have checked, we have found complete agreement with earlier results based either on Random Matrix Theory or the supersymmetric technique. The replica method constitutes a new and independent derivation of these results.

With the expansions of the partition function at hand we have also derived the small-mass and largemass expansions of the partially quenched chiral condensate. In all cases we have checked there is again complete agreement with earlier analytical predictions based on the two other methods.

We have here restricted ourselves to small-mass and large-mass series solutions only because they provide the simplest framework in which to employ the replica method. This is not an inherent restriction, though. It is thus an open challenge to extend the method beyond these two series expansions.

ACKNOWLEDGEMENT:

We thank M. Lüscher and H.B. Nielsen for an early conversation. This work was supported in part by EU TMR grant no. ERBFMRXCT97-0122. 


\section{References}

[1] H. Leutwyler and A. Smilga, Phys. Rev. D46 (1992) 5607.

[2] A. Smilga and J.J.M. Verbaarschot, Phys. Rev. D51 (1995) 829.

[3] E.V. Shuryak and J.J.M. Verbaarschot, Nucl. Phys. A560 (1993) 306.

[4] J.J.M. Verbaarschot and I. Zahed, Phys. Rev. Lett. 70 (1993) 3852; Phys. Rev. Lett. 73 (1994) 2288.

J.J.M. Verbaarschot, Nucl. Phys. B426 (1994) 559; Phys. Rev. Lett. 72 (1994) 2531.

[5] G. Akemann, P.H. Damgaard, U. Magnea and S. Nishigaki, Nucl. Phys. B487 (1997) 721.

P.H. Damgaard and S.M. Nishigaki, Nucl. Phys. B518 (1998) 495.

M.K. Şener and J.J.M. Verbaarschot, Phys. Rev. Lett. 81 (1998) 248.

[6] P.H. Damgaard, Phys. Lett. B424 (1998) 322.

G. Akemann and P.H. Damgaard, Nucl. Phys. B519 (1998) 682; Phys. Lett. B432 (1998) 390; hep-th/9910190.

[7] J.C. Osborn, D. Toublan and J.J.M. Verbaarschot, Nucl. Phys. B540 (1999) 317.

P.H. Damgaard, J.C. Osborn, D. Toublan and J.J.M. Verbaarschot, Nucl. Phys. B547 (1999) 305.

D. Toublan and J.J.M. Verbaarschot, hep-th/9904199.

[8] J.J.M. Verbaarschot and M.R. Zirnbauer, J. Phys. A18 (1985) 1093.

[9] A. Kamenev and M. Mezard, cond-mat/9901110, cond-mat/9903001.

I.V. Yurkevich and I.V. Lerner, cond-mat/9903025.

M.R. Zirnbauer, cond-mat/9903338.

E. Kanzieper, cond-mat/9908130.

[10] J.J.M. Verbaarschot, Phys. Lett. B368 (1996) 137.

[11] P.H. Damgaard, R.G. Edwards, U.M. Heller and R. Narayanan, hep-lat/9907016; hep-lat/9909009.

P. Hernandez, K. Jansen and L. Lellouch, hep-lat/9907022; hep-lat/9909026.

[12] R. Brower, P. Rossi and C.-I. Tan, Nucl. Phys. B190[FS3] (1981) 699.

A.D. Jackson, M.K. Şener and J.J.M. Verbaarschot, Phys. Lett. B387 (1996) 355.

[13] D.J. Gross and M.J. Newman, Nucl. Phys. B380 (1992) 168.

[14] A. Mironov, A. Morozov and G.W. Semenoff, Int. J. Mod. Phys. A11 (1996) 5031.

[15] B. de Wit and G. 't Hooft, Phys. Lett. B69 (1977) 61.

[16] I. Bars, J. Math. Phys. 21 (1980) 2678.

[17] J.J.M. Verbaarschot, Phys. Lett. B329 (1994) 351.

[18] J. Verbaarschot, Nato Lectures Cambridge 1997 on "Confinement, Duality, and Nonperturbative Aspects of QCD" p. 343-378, hep-th/9710114.

T. Akuzawa and M. Wadati, J. Phys. Soc. Jap. 67 (1998) 2151.

[19] P.H. Damgaard, Phys. Lett. B425 (1998) 151. 
[20] T. Wilke, T. Guhr and T. Wettig, Phys. Rev. D57 (1998) 6486.

[21] J. van der Leur, solv-int/9909028. 